Supporting Information

\title{
Synthesis of Polyporous Ion-sieve and Its Application for Selective Recovery of Lithium from Geothermal Water
}

Hongyu Lin ${ }^{1}$, Xiaoping Yu *,1, Mingli Li ${ }^{2}$, Ji Duo ${ }^{2}$, Yafei Guo ${ }^{1}$, Tianlong Deng ${ }^{*}, 1$

${ }^{1}$ Tianjin Key Laboratory of Brine Chemical Engineering and Resource Eco-utilization, Tianjin University of Science and Technology, Tianjin 300457, China

${ }^{2}$ Central Laboratory of Geological Mineral Exploration and Development Bureau of Tibet Autonomous Region, Tibet 850033, China

${ }^{*}$ Corresponding author. E-mail: yuxiaoping@tust.edu.cn; tldeng@tust.edu.cn. 


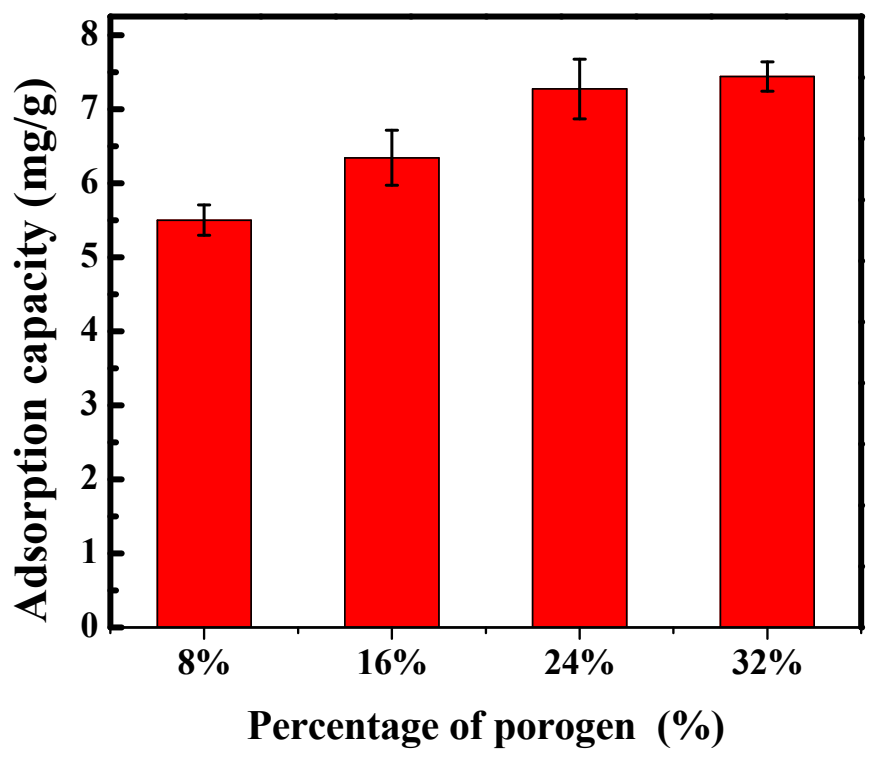

Figure S1. Effect of the adding amount of PEG-6000 on the adsorption capacity of $\mathrm{Li}^{+}$. 


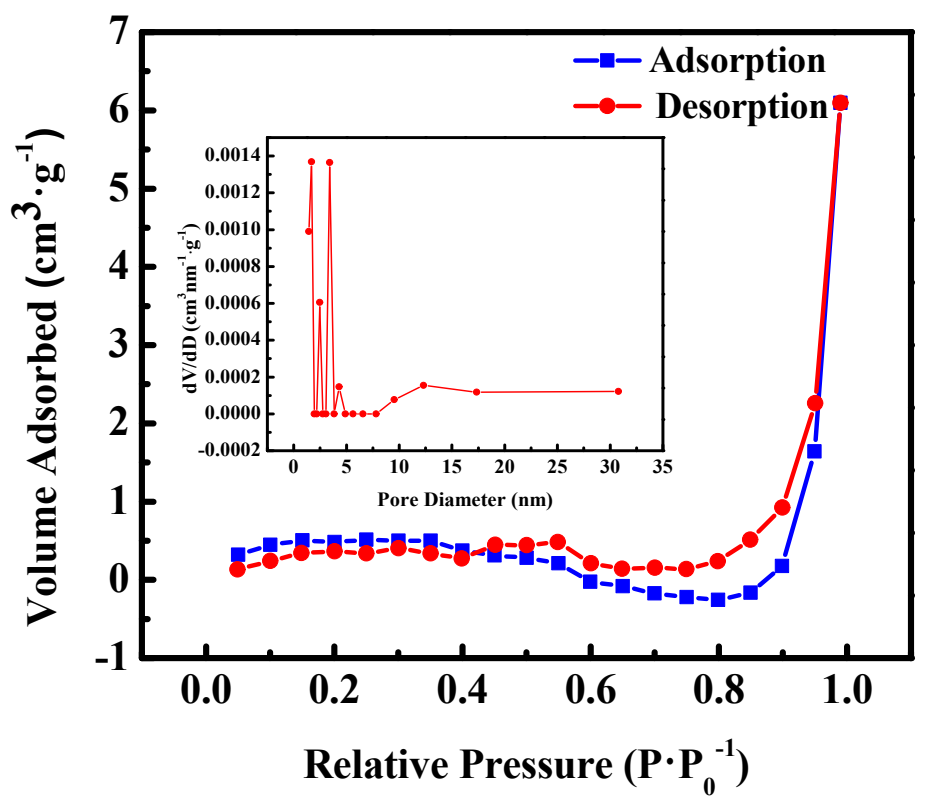

Figure S2. Result on the BET test of the developed material. 